\section{Cuidado resolutivo de paracoccidioidomicose em uma Unidade Básica de Saúde. Relato de caso}

\author{
Resolutive care of paracoccidioidomycosis in a Basic Health Unit. \\ Case report
}

\author{
Resolución de paracoccidioidomicosis en una Unidad Básica de \\ Salud. Relato de caso
}

\section{Resumo}

A paracoccidioidomicose é uma micose sistêmica endêmica nas regiões tropicais da América do Sul, sendo comum no Brasil. É causada pelo fungo Paracoccidioides brasiliensis, que infecta o homem pela via respiratória por meio de inalação. Após a sintomatologia pulmonar, por disseminação hematogênica, pode acometer vários órgãos e sistemas. Uma das dificuldades no diagnóstico dessa doença se deve à sua semelhança clínica com a tuberculose. Clinicamente, a doença pode apresentar-se nas formas crônica e aguda/subaguda. Constitui-se em um importante problema de saúde pública, pois é uma doença incapacitante, podendo levar o portador ao óbito. Descreve-se, neste artigo, um caso de paracoccidioidomicose em indivíduo do sexo masculino, cuja resolutividade do cuidado pela equipe da Unidade Básica de Saúde (UBS) foi fundamental para o sucesso do tratamento e para o bom prognóstico.

Palavras-chave: Paracoccidioidomicose; Estomatite; Micoses; Tuberculose
Maria Cristina Almeida de Souza ${ }^{a}$, Edsneider Rocha Pires de Souza ${ }^{a}$ Paula Pitta de Resende Côrtes ${ }^{b}$ João Carlos de Souza Côrtes Júnior ${ }^{b}$ Sandra Maria Barroso Werneck Vilagra ${ }^{\mathrm{a}}$ Elisa Maria Amorim da Costa ${ }^{a}$

\footnotetext{
a Universidade de Vassouras. Vassouras, RJ, Brasil. mcas.souza@uol.com.br (Autor correpondente); edsneidermed@yahoo.com.br; sm.vilagra@uol.com.br; elisamacosta.emac@gmail.com

b Universidade de Vassouras. Universidade Federal do Estado do Rio de Janeiro (UNIRIO). Rio de Janeiro, RJ, Brasil. paulapitta@yahoo.com.br; joaocortes@yahoo.com.br
}

Como citar: Souza MCA, Souza ERP, Côrtes PPR, Côrtes Júnior JCS, Vilagra SMBW, Costa EMA. Cuidado resolutivo de paracoccidioidomicose em uma Unidade Básica de Saúde. Relato de caso. Rev Bras Med Fam Comunidade. 2018;13(40):1-7. http://dx.doi.org/10.5712/rbmfc13(40)1713
Fonte de financiamento: declaram não haver. Parecer CEP: não se aplica. Conflito de interesses: declaram não haver. Procedência e revisão por pares: revisado por pares. Recebido em: 08/03/2018. Aprovado em: 18/11/2018. 


\section{Resumen}

La paracoccidioidomicosis es una micosis sistémica endémica en las regiones tropicales de América del Sur, siendo común en Brasil. Es causada por el hongo Paracoccidiodes brasiliensis, que infecta al hombre por la vía respiratoria por inhalación. Después de la sintomatología pulmonar, por diseminación hematógena, puede acometer varios órganos y sistemas. Una de las dificultades en el diagnóstico de esta enfermedad se debe a su semejanza clínica con la tuberculosis. Clínicamente, la enfermedad puede presentarse en las formas crónica y aguda/subaguda. Se constituye un importante problema de salud pública debido a su potencial incapacitante, pudiendo llevar al portador al óbito. Se describe en este artículo un caso de paracoccidioidomicosis en usuario del sexo masculino, cuya resolución del cuidado por el equipo de la Unidad Básica de Salud (UBS) ha sido fundamental para el éxito del tratamiento y para el buen pronóstico.

Palabras clave: Paracoccidioidomicosis; Estomatitis; Micosis; Tuberculosis

\section{Introdução}

A paracoccidioidomicose (PCM), blastomicose sul-americana ou moléstia de Lutz-Splendore e Almeida, é uma micose sistêmica causada pelo fungo Paracoccidioides brasiliensis, cujo nicho ecológico é o solo. ${ }^{1-6}$

Acomete qualquer tecido ou órgão, embora seja o pulmão o órgão mais frequentemente afetado. Clinicamente, pode apresentar-se nas formas crônica (tipo adulta) e aguda/subaguda (tipo juvenil). ${ }^{1,3,5,6}$

A forma aguda/subaguda representa cerca de $3-5 \%$ dos casos, sendo a forma clínica mais prevalente na infância, podendo eventualmente acometer adultos com mais de 30 (até os 40) anos de idade. Os principais sintomas são linfonodomegalias superficiais e profundas, com supuração de massa ganglionar, sintomas digestivos, cutâneos, osteoarticulares, hepatoesplenomegalia, anemia, febre e caquexia. Nessa fase é raro o comprometimento pulmonar. ${ }^{5}$

A fase crônica - que pode ser unifocal (acomete um único órgão) ou multifocal (vários órgãos são afetados) - é a forma observada em cerca de $90 \%$ dos casos. Nesta fase, geralmente são acometidos adultos com 30 a 60 anos de idade. A doença instala-se de forma mais lenta, com duração da sintomatologia acima de quatro a seis meses e, por vezes, acima de um ano. Em alguns casos, a instalação é silenciosa e a doença é flagrada por exame solicitado para fins trabalhistas ou check-up. ${ }^{7}$ Envolvimento pulmonar nesta fase é comum e mimetiza a tuberculose. Radiograficamente, observam-se lesões nodulares, intersticiais ou cavitárias, mas que diferem da tuberculose e da histoplasmose devido à tendência de localização dos infiltrados nos campos pulmonares médios e inferiores, em vez dos ápices. ${ }^{4,5}$

A paracoccidioidomicose se desenvolve após a inalação de esporos do microorganismo existentes no meio ambiente. Após a sintomatologia pulmonar, o fungo pode atingir vários órgãos e sistemas através da disseminação hematogênica. ${ }^{4,8}$

A dificuldade no diagnóstico da PCM é devido aos sintomas serem semelhantes aos da tuberculose (TB). Nas fases iniciais da enfermidade, os sinais clínicos e radiológicos nem sempre permitem clara distinção entre a PCM e a TB. Os principais sintomas da forma unifocal da PCM são fraqueza, emagrecimento, febre, tosse, dispneia, infiltrado reticulonodular e hipertransparência distal bibasal. Já na forma multifocal da PCM, a doença acomete outros órgãos além do pulmão, como pele, mucosa oral, mucosas da faringe, laringe e o ápice dos dentes, causando dor durante a mastigação, sialorreia e odinofagia. ${ }^{5}$ 
É uma doença endêmica nas regiões tropicais da América do Sul, sendo comum no Brasil. Sua incidência é maior entre os homens, especialmente aqueles na faixa etária de entre 30 a 50 anos. ${ }^{9} \mathrm{O}$ motivo da ocorrência desigual entre os sexos está possivelmente relacionado a efeitos inibitórios dos estrogênios sobre o crescimento do organismo e não somente à maior exposição ambiental. ${ }^{4,5}$

A anamnese adequada, o exame físico detalhado, análises laboratoriais e radiografias são importantes para o diagnóstico definitivo. O diagnóstico definitivo (padrão ouro) acontece pelo achado do fungo em espécimes clínicos ou na biópsia tecidual. Não existe, até o momento, prevenção específica para a paracoccidioidomicose, doença que não faz parte da relação de notificação compulsória.

Deve-se tratar os portadores a fim de evitar o desenvolvimento da doença e o surgimento de complicações, sempre considerando a disponibilidade do acesso à medicação durante o tratamento e a adoção de medidas que melhorem as condições gerais do doente. ${ }^{10} \mathrm{O}$ tratamento é usualmente feito com os fármacos sulfametoxazol-trimetoprim, anfotericina $\mathrm{B}$, drogas antifúngicas, sulfamídicos e compostos azólicos, isolados ou associados. ${ }^{3,9}$ Nem todos, contudo, integram a Relação Nacional de Medicamentos (RENAME) ou mesmo a REMUME (Relação Municipal de Medicamentos).

Justifica-se assim, a necessidade de que a equipe da Estratégia Saúde da Família, porta de entrada preferencial ao Sistema Único de Saúde (SUS), esteja qualificada e atualizada para diagnosticar a doença, bem como prestar os cuidados necessários ao seu manejo.

O Brasil é um dos países de maior endemicidade da paracoccidioidomicose, que representa um importante problema de saúde pública, devido ao seu alto potencial de morbidade e ao número de mortes prematuras que provoca, especialmente entre trabalhadores rurais do sexo masculino.

O objetivo deste artigo é descrever um caso de paracoccidioidomicose em usuário do sexo masculino, cuja resolutividade do cuidado pela equipe da USF foi fundamental para o sucesso do tratamento e bom prognóstico.

\section{Detalhamento do caso}

Paciente do sexo masculino, 27 anos, leucoderma, auxiliar administrativo, compareceu à Unidade Básica de Saúde (UBS) apresentando como queixa principal a presença de lesões em alto-relevo, eritematosas, nas regiões torácica (Figura 1A e 1B) e retroauricular (Figura 1C e 1D).

$\mathrm{Na}$ anamnese, relatou ao médico de família e comunidade (MFC) que as lesões haviam surgido há cerca de 1 mês. E que, 7 dias após o início, havia procurado por cuidados no serviço de pronto atendimento hospitalar, cujo médico lhe prescreveu um antifúngico tópico (miconazol gel 20 miligramas), com orientações para aplicação 5 vezes ao dia nas lesão durante 7 dias.

Decorrido o prazo, como não houve constatação de remissão das lesões, retornou ao serviço de saúde. Foi-lhe prescrito então corticoide para uso tópico (cetoconazol $20 \mathrm{mg}+$ dipropionato de betametasona 0,5mg), com orientações para aplicação nos locais da lesão, alternadamente ao antifúngico, de modo que uma vez ao dia o produto aplicado fosse o antifúngico e, duas vezes, o corticoide, totalizando aplicação de produtos três vezes ao dia durante 7 dias. Um retorno após este período foi-lhe solicitado pelo médico. Não foi prescrito medicamento para uso interno. 


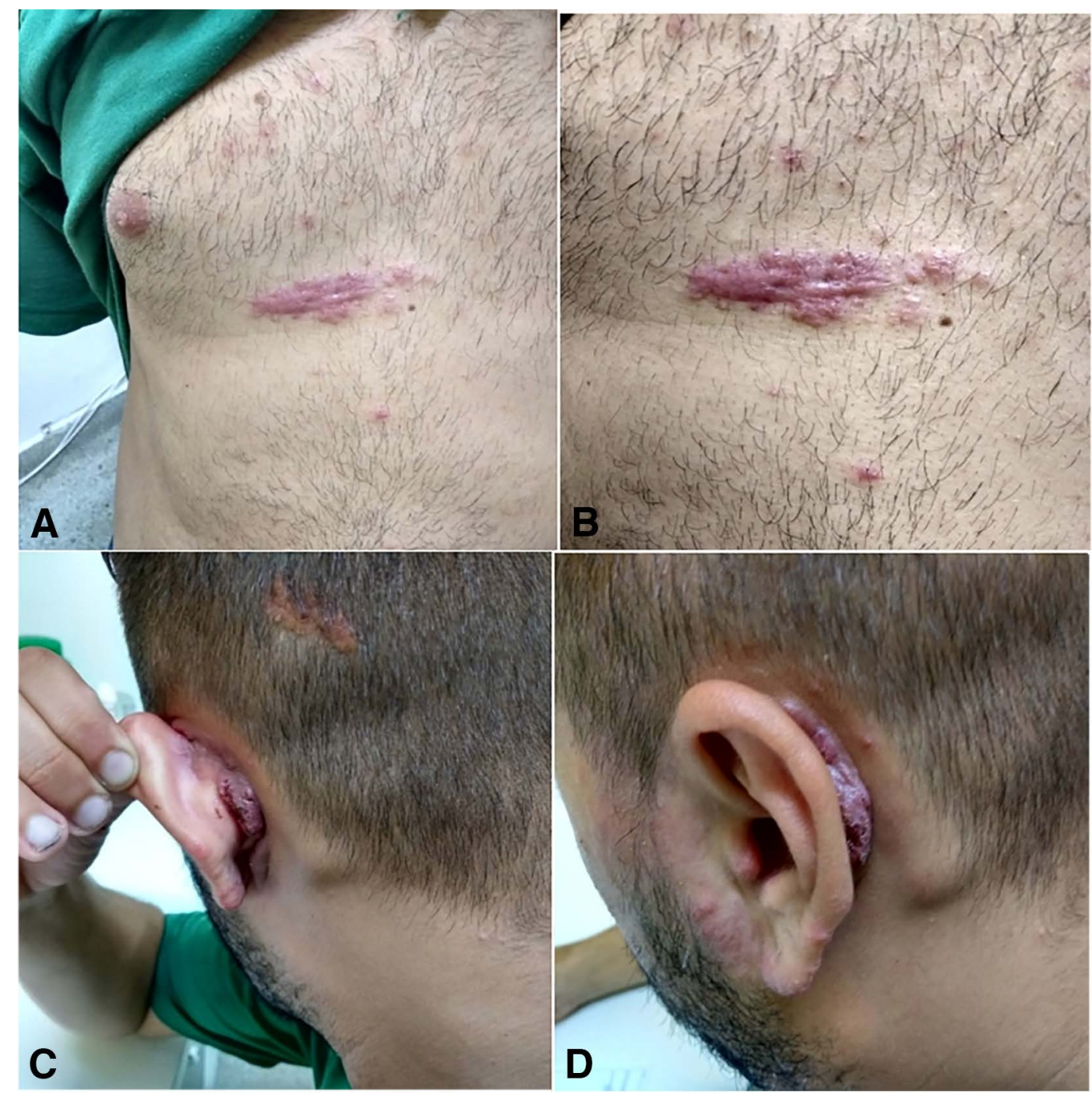

Figura 1. A e B. Lesão na região torácica. C e D. Lesão na região retroauricular.

Vencido o prazo, e sem constatação de melhoras, o paciente relatou que optou por comparecer à UBS. Confidenciou que a agente comunitária de saúde (ACS) vinha insistindo para que se consultasse com o médico da UBS, mas que ele estava relutante até então.

Negou histórico de febre, inaptência, limitação funcional, prurido, ardência ou dor. Teste rápido antiHIV realizado na UBS deu resultado negativo. Hiperemia foi observada nas regiões afetadas. Ao exame clínico, o MFC observou paciente normocorado, com pressão arterial $120 \times 80 \mathrm{mmHg}$, valores de glicemia capilar: $89 \mathrm{mg} / \mathrm{dl}$. Sem relato de ingestão de qualquer medicação de uso contínuo.

Foi constatada pelo MFC a presença de prurido na lesão na região retroauricular e a existência de gânglios na região cervical e axilar. Mucosas oral, nasal e faríngea: sem alterações. Paciente negou histórico de trabalhado rural, mas relatou que 5 dias antes do surgimento das lesões, havia feito uma limpeza em um galpão abandonado no quintal da residência, no qual havia muita serragem, restos de madeira apodrecida, tijolos antigos e fezes de pombos. 
A hipótese diagnóstica foi de PCM, que é endêmica no Brasil. Como não havia expectoração, descartou-se, naquele momento, possibilidade de tuberculose. A dificuldade no diagnóstico clínico dessa doença é devido sua semelhança clínica com a tuberculose (TB), porém com clara distinção no diagnóstico laboratorial, já que as duas afecções possuem agentes etiológicos bem distintos. ${ }^{5}$

Atendendo aos protocolos do município, que tem cobertura populacional da Estratégia Saúde da Família é de $100 \%$ e Rede de Atenção à Saúde sistematizada, o MFC referenciou o usuário ao serviço de média complexidade - ambulatório de cirurgia - para realização de biópsia. Solicitou também exames complementares como radiografia de tórax, ultrassonografia abdominal, hemograma (função hepática), cujos resultados contribuem para o diagnóstico diferencial. O paciente foi orientado a suspender a aplicação dos produtos tópicos até o resultado da biópsia e a higienizar cuidadosamente o local.

O exame de sangue revelou normalidade de função hepática e demais taxas. O ultrassom abdominal não evidenciou alteração, contribuindo para descartar definitivamente a hipótese de tuberculose. A radiografia de tórax apresentou imagem sugestiva de discreto velamento parcial em ambos os pulmões. O laudo da biópsia confirmou o diagnóstico de PMC. No caso aqui relatado, a elaboração do diagnóstico não demandou por contatos ou contribuições de especialistas, tendo sido realizado pelo MFC.

Foi então prescrito uso sistêmico do antifúngico itraconazol 100 miligramas, 1 cápsula a cada 12 horas por 4 semanas (dose de ataque), quando o paciente teve uma consulta de retorno previamente agendada. Paciente relatou ser alérgico à sulfa. Ao ACS foi solicitado que, até a data da citada consulta, durante as visitas domiciliares (VD), perguntasse ao paciente sobre a evolução das lesões e informasse ao MFC para acompanhamento e registro no prontuário.

Decorrido as 4 semanas da consulta inicial, o paciente retornou à UBS e o MFC constatou significativa remissão das lesões (Figura 2A e 2B), descartando uso de qualquer outra medicação adicional.

Paciente foi orientado a reduzir o uso da medicação para 1 comprimido a cada 24 horas pelos 6 meses seguintes (dose de manutenção). Foi ratificada a relevância de consultas semestrais na UBS. O prognóstico revelou-se bom devido à adequada terapia instituída.
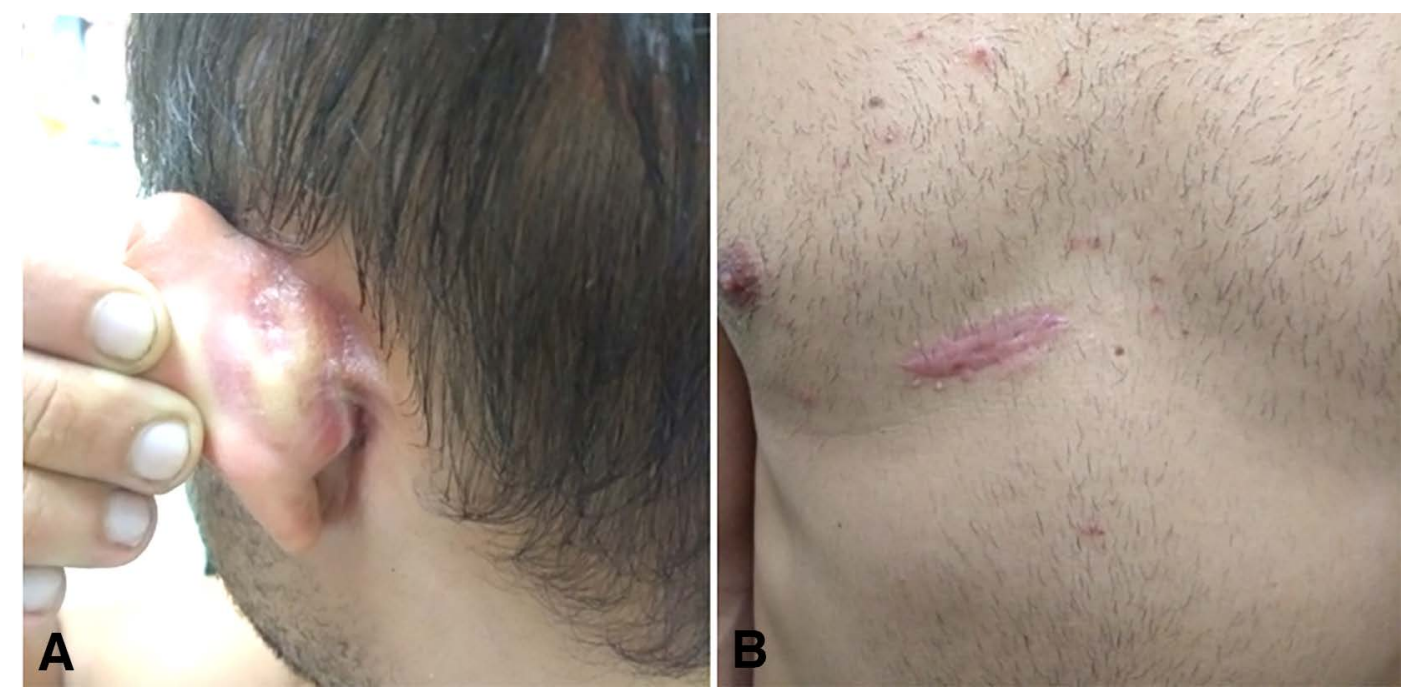

Figura 2. A e B. Remissão das lesões. 


\section{Discussão}

APCM é a maior causadora de morte em consequência de micoses sistêmicas no Brasil. ${ }^{4,10}$ Tendo em vista que a principal porta de entrada ao Sistema Único de Saúde (SUS) é a UBS, torna-se imprescindível que as equipes de saúde que nelas atuam estejam atualizadas para prestarem um cuidado resolutivo ao usuário destes serviços.

No caso aqui relatado, a resolutividade do cuidado coube à equipe da UBS, embora o paciente já tivesse se consultado, sem sucesso, com outros profissionais, em uma clara demonstração de que o monitoramento do tratamento constitui um diferencial dos profissionais que atuam na Atenção Primária à Saúde (APS).

Cabe ressaltar que a competência clínica da equipe - materializada por meio do emprego de seus conhecimentos, habilidades e atitudes - foi um diferencial para a resolutividade do cuidado prestado ao usuário e contribuiu para evidenciar a expertise de seu processo de trabalho. Trata-se de uma equipe que norteia seu cotidiano profissional pela premissa de que é capaz de resolver $80 \%$ das demandas da população adscrita à UBS, principalmente pela sistemática participação de seus integrantes em atividades de educação permanente, periodicamente ofertadas pela gestão municipal.

O vínculo do paciente com o ACS, que sabidamente conhecia seu estilo e hábitos de vida, foi fundamental para o adequado cuidado, pois provavelmente a insistência do ACS para que o paciente procurasse o médico da UBS representou um fator importante na decisão.

Embora a tosse produtiva e a febre - sinais frequentes da doença - estivem ausentes, a hipótese diagnóstica da paracoccidioidomicose foi levantada devido presença de lesões na pele, ao envolvimento ganglionar, à localização das lesões na região superior do corpo - sinais característicos da doença ${ }^{4} \mathrm{e}$ também ao relato pelo paciente, do manuseio de material empoeirado e em decomposição em um local abandonado, a exemplo do informado na literatura - de que o contágio ocorre pela inalação do aerossol formado pela poeira e outros componentes do solo, entre eles, os fungos do gênero Paracoccidioides, no revolvimento de terra. ${ }^{11}$ Surto de paracoccidioidomicose após desmatamento e remoção massiva de terra durante a construção de uma rodovia no Rio de Janeiro, Brasil foi relatado na literatura. ${ }^{12}$

A confirmação da suspeita foi possível com os resultados dos exames laboratoriais e da biópsia, atendendo assim ao preconizado pelo Ministério da Saúde sobre a importância destes laudos para o diagnóstico diferencial, evitando subnotificações. ${ }^{1}$

O tratamento do caso descrito com itraconazol, assim como relatado na literatura, ${ }^{5,10}$ foi realizado em duas fases: a de ataque e a de manutenção. Na primeira, o objetivo foi promover, além do controle imediato dos sinais e sintomas, a redução da carga parasitária, visando à recuperação da imunidade celular do hospedeiro. A segunda fase, de manutenção, foi instituída para reduzir o risco de recorrência da doença.

Justifica-se a opção pelo uso do derivado azólico itraconazol como droga de primeira escolha, devido a sua comprovada eficácia e tolerabilidade no tratamento da parcoccidiodomicose,${ }^{10}$ decorrente da inibição da síntese do ergosterol, comprometendo a permeabilidade da membrana do fungo. Diferentemente de autores que prescrevem sulfonamidas para a terapia da paracoccidioidomicose, devido à alergia do paciente a este fármaco e seus derivados, o MFC optou pelo itraconazol, ainda que não fosse fornecido gratuitamente pelo SUS. Paciente afirmou ter condições financeiras para adquirir o fármaco. 
O uso do cetoconazol, ainda que efetivo, foi descartado devido aos efeitos colaterais e à ocorrência de mais recidivas. Há pouca experiência com o fluconazol, que ainda não é o medicamento de primeira escolha. A anfotericina $B$, ainda que indicada para o tratamento de PCM, não foi prescrita devido a sua nefrotoxicidade e aos seus efeitos colaterais. Quanto às sulfas, embora se mostrem medicamentos eficazes no tratamento da paracoccidioidomicose e sejam fornecidas pelo SUS, apresentam a desvantagem de terem de ser administradas várias vezes ao dia e por um longo período. .,10 $^{-10}$

O conhecimento sobre a paracoccidioidomicose ainda requer desvendar muitos dos aspectos relevantes da biologia do fungo, sua fisiopatologia, métodos diagnósticos menos intervencionistas, terapêutica mais curta e controle de cura adequado..$^{13}$ Lamentavelmente, a PCM ainda tem sua incidência e prevalência subestimadas devido à ausência de notificação em várias Unidades da Federação. ${ }^{7}$

\section{Referências}

1. Brasil. Ministério da Saúde. Doenças infecciosas e parasitárias. Guia de Bolso. 8ª ed. Brasília: Ministério da Saúde; 2010 [acesso 2018 Fev 12]. Disponível em: http://bvsms.saude.gov.br/bvs/publicacoes/doencas_infecciosas_parasitaria_guia_bolso.pdf

2. Prohmann CM, Dantas CA, Silva LB, Gatti RF, Machado TM, Antônio JR. Paracoccidioidomicose com acometimento ganglionar e mucoso - relato de caso. Rev SPDV. 2017;75(3):283-7 [acesso 2018 Fev 12]. Disponível em https://revista.spdv.com.pt/index.php/spdv/article/ view/818

3. Souza NE, Afonso EO, Caldeira CA, Werneck FA, Boechat TO. Paracoccidioidomicose em criança com hipereosinofilia: relato de caso. Rev Saúde. 2017;8(1):33-6 [acesso 2018 Fev 15]. Disponível em: http://editora.universidadedevassouras.edu.br/index.php/RS/article/ view/308

4. Kauffman CA. Paracoccidioidomicose. In: Goldman L, Ausiello D. Ceccil-Tratado de Medicina Interna. 23를. edão Paulo: Elsevier; 2009. p. 2708-9.

5. Sanches LC, Faria MCI. A importância do diagnóstico diferencial entre a paracoccidioidomicose (PCM) e tuberculose (TB). Rev Unigá Review 2014;20(1):76-80 [acesso 2018 Nov 18]. Disponível em: https://www.mastereditora.com.br/periodico/20141001_084211.pdf

6. Toledo GL, Marzola C, Toledo Filho JL, Capelari MM. Blastomicose sul americana - apresentação de caso clínico tratado com associação de inidazóis sistêmico e tópico. Rev Port Estomatol Med Dent Cir Maxilfac. 2011;52(2):83-8 [acesso 2018 Fev 16].http://dx.doi.org/10.1016/ S1646-2890(11)70016-4

7. Shikanai-Yasuda MA, Mendes RP, Colombo AL, Telles FQ, Kono A, Paniago AMM, et al. Brazilian guidelines for the clinical management of paracoccidioidomycosis. II Consenso em Paracoccidioidomicose. Epidemiol Serv Saúde. 2018;27(núm. esp.):e0500001 [acesso 2018 Nov 11]. http://dx.doi.org/10.5123/S1679-49742018000500001

8. Gavazzoni MFR, Azulay DR, Azulay RD. Paracoccidioidomicose (blastomicose sul-americana, micose de Lutz). In: Azulay RD, Azulay DR, Azulay-Abulafaia L. Dermatologia. 6ª ed. Rio de Janeiro: Guanabara Koogan, 2013.

9. Wanke B, Aidê MA. Paracoccidioidomicose. J Bras Pneumol. 2009;35(12):1245-9 [acesso 2018 Fev 15]. http://dx.doi.org/10.1590/ S1806-37132009001200013

10. Uchimura MH, Francisco RS, Carmo HMO, Carvalho CM. Paracoccidioidomicose: um panorama da doença na amazônia. Braz J Surg Clin Res. 2018;21(3):113-8 [acesso 2018 Fev 15]. Disponível em: https://www.mastereditora.com.br/periodico/20180204_153017.pdf

11. Ricci CD, Evangelista C, Thomaz BCA, Silva MV, Barbo MLP. Paracoccidioidomicose: forma crônica cutânea. Rev Fac Ciênc Méd Sorocaba. 2018;20(1):51-4 [acesso 2018 Nov 18]. http://dx.doi.org/10.23925/1984-4840.2018v20i1a12

12. Valle AC, Marques PM, Almeida-Paes R, Romão AR, Lazéra M, Wanke B, et al. Paracoccidioidomycosis after highway construction, Rio de Janeiro, Brazil. Emerg Infect Dis. 2017;23(11):1917-9 [acesso 2018 Fev 26]. Disponível em: https://wwwnc.cdc.gov/eid/ article/23/11/17-0934_article

13. Ambrósio AVA, Camelo CCS, Barbosa CV, Tomazatti FG, Brazões FAZ, Veloso JM, et al. Paracoccidioidomicose (doença de LutzSplendore-Almeida): propedêutica complementar, diagnóstico diferencial, controle de cura. Rev Med Minas Gerais. 2014;24(1):81-92 [acesso 2018 Nov 11]. Disponível em: http://www.rmmg.org/artigo/detalhes/606 